\title{
Physiotherapy clinical education at a South African university
}

\author{
V Chetty, PhD; S Maddocks, MPhysio; S Cobbing, PhD; N Pefile, MSc, MedSc (Rehabilitation); T Govender, BPhysio; S Shah, BPhysio; H Kaja, BPhysio; \\ R Chetty, BPhysio; M Naidoo, BPhysio; S Mabika, BPhysio; N Mnguni, BPhysio; T Ngubane, BPhysio; F Mthethwa, BPhysio
}

Discipline of Physiotherapy, School of Health Sciences, University of KwaZulu-Natal, Durban, South Africa

Corresponding author: V Chetty (chettyve@ukzn.ac.za)

\begin{abstract}
Background. Clinical education for physiotherapists forms a vital part of undergraduate programmes and equips students with competencies to practise autonomously as qualified health practitioners. However, disparities are evident in approaches to clinical education.

Objective. To explore the perceptions of physiotherapy students, community-service physiotherapists and physiotherapy clinical supervisors regarding the clinical education framework at a tertiary institution in South Africa in order to understand preparedness of students for practice.

Methods. A case study approach with two focus group discussions with students and interviews with community physiotherapists and clinical supervisors was employed. Data were analysed and categorised into key themes and sub-themes.

Results. Five themes emerged from triangulation of data from the three groups: preparedness for professional practice, institutional barriers, curriculum disputes, personal factors and recommendations for physiotherapy clinical education. Students felt inadequately prepared owing to a perceived lack of exposure to certain aspects of physiotherapy, while community therapists believed that reflection on the undergraduate programme after qualifying contributed to their adequate preparation. Clinical supervisors supposed that students would benefit from actively engaging with teaching and learning opportunities, and clinical personnel collaboration was seen as key to facilitate a continuum in clinical education from classroom to healthcare setting. Conclusion. Participants reported that the existing curriculum structure may need to be revisited to address various issues, while holistic collaboration between students, supervisors and clinical personnel is imperative to create a cohesive learning environment.
\end{abstract}

Afr J Health Professions Educ 2018;10(1):13-18. DOI:10.7196/AJHPE.2018.v10i1.987

Physiotherapy is a health profession that is focused on the rehabilitation of individuals faced with impairments, activity limitations and/or disabilities that affect daily life. Furthermore, physiotherapists promote quality of life through identification of environmental and social barriers and promotion of health and wellbeing. ${ }^{[1]}$ Becoming a registered physiotherapist in South Africa (SA) requires graduating from an undergraduate training programme at one of eight universities and successfully completing 1 year as a remunerated supervised community-service physiotherapist. ${ }^{[2]}$

The duration of the SA undergraduate physiotherapy programme is 4 years, leading to a Bachelor of Science in Physiotherapy degree. ${ }^{[2]}$ The university where this study took place offers a physiotherapy programme with an intake of $\sim 50$ students in the first year. In the second year of undergraduate training, students are exposed to clinical learning platforms, but are merely observers at this level. In the third year, students begin clinical rotations in groups of 6 or 7 for periods of 5 weeks -4 blocks per year. The clinical blocks cover cardiopulmonary, neurological and neuromuscular conditions and community rehabilitation, including health promotion and awareness. During the third year, students assess and treat patients individually under the supervision of clinical supervisors and physiotherapists employed at clinical sites. Students are subjected to both formative and summative assessments, including bedside clinical examinations at the culmination of each block. The final year of the programme is a reflection of the third-level framework but evolves into specific areas of practice, such as intensive care rehabilitation and orthopaedic mobilisation. ${ }^{[3]}$ Students are also expected to manage patients independently and demonstrate clinical reasoning, including diagnosis and prognosis of patients' conditions to optimise rehabilitation. During their final year, students undergo a summative externally moderated examination at the clinical placement areas. Students in their third and fourth years are exposed to clinical practice in primary healthcare settings, as well as various public healthcare contexts, spanning quaternary-, tertiary- and district-level hospitals. These students are supervised and assessed by clinical educators, who are academic staff or university-employed physiotherapy clinicians. They are referred to as clinical supervisors for the purpose of this article. Two or sometimes three clinical supervisors are responsible for clinical training of physiotherapy students per clinical block. Supervisors facilitate group work through case presentations and problem-based learning and use individual bedside teaching as core strategies of learning. There is at least one clinical supervisor per day to supervise the 6 or 7 students per clinical block. Supervision time varies from 1 hour of case presentations with all students to supervising students managing patients for 45 minutes - 1 hour at least once during a block. The nature of clinical supervision is consistent throughout the clinical blocks. The role of clinical supervisors is essential in the co-creation of knowledge and facilitation of learning within the physiotherapy programme. ${ }^{[4]}$

Clinical education is essential to prepare undergraduate physiotherapy students to gain profession-specific knowledge, develop technical skills and become socially and ethically competent to practise independently. ${ }^{[5-8]}$ In SA, these are governed by the universities' graduate competencies framework. Health science students should demonstrate adeptness in seven key roles, i.e. as practitioner, communicator, collaborator, leader, scholar, health advocate and professional. ${ }^{[9]}$ Although clinical education is fundamental for the preparation of students to practise autonomously, little evidence exists on the approaches to deliver an ideal model for training of the physiotherapy 
student. ${ }^{[5]}$ A review of clinical education models was conducted in 2007, which analysed a milieu of models in global contexts. ${ }^{[7]}$ These included the one-educator-to-one-student model, one-educator-to-multiple-students model, multiple-educators-to-one-student model, multiple-educators-tomultiple-students and/or non-discipline-specific-educator model, and finally student-as-educator model. The review found that no model proved to be superior to another and that benefits and inhibitors influencing the various models were used internationally in tertiary institutions. ${ }^{[7]}$ Furthermore, a gap exists regarding a model to guide clinical education in the current SA context and approaches to address discrepancies in tertiary clinical training. This study aimed to explore the views of current students, past students who were in their community-service year of practice, as well as clinical supervisors - to understand the landscape for co-operative construction of the clinical education platform at the tertiary institution of research interest in this study. Another objective was to contribute to the development of an integrated teaching and learning model of clinical education within the current study context to inform further enquiry and have a positive influence on the SA health science education.

\section{Methods}

A qualitative explorative case study approach allowed for investigation of the clinical education component of a physiotherapy programme at a tertiary institution in SA. ${ }^{[10]}$ With this approach, real-life phenomena could be studied, as experienced by students, community-service physiotherapists and clinical supervisors in a resource-limited higher education context. ${ }^{[10,11]}$ An holistic single case study with triangulation of data from the students, community physiotherapists and clinical supervisors was used to understand the clinical education experience at the university and its collaborating clinical learning platforms. ${ }^{[1]}$

\section{Ethical approval}

The study was approved by the Humanities and Social Sciences Research Ethics Committee at the University of KwaZulu-Natal in SA (ref. no. HSS/1124/016U). Permission was also obtained from all designated authorities, including the academic leader of the university's physiotherapy department.

\section{Participant enlistment}

The following were recruited through purposive maximum variation sampling: 22 final-year physiotherapy students from a class of 50; 9 community-service physiotherapists from various clinical settings, including rural, urban and semi-rural settings; and 9 of 12 physiotherapy clinical supervisors responsible for clinical education and supervision of students. Maximum variation sampling for the current case study approach allowed for a wide range of views, including current students, communityservice physiotherapists, i.e. physiotherapy students who completed their undergraduate degree at the study setting the previous year, as well as clinical supervisors. ${ }^{[12]}$ All participants signed informed consent forms to participate voluntarily in the study. No incentives were offered. Pseudonyms were used to annotate quotes from participants.

\section{Data collection}

Two focus group ( $n=12$ and $n=10$ ) discussions were conducted with the final-year students at the institution to allow an open discussion with each other and to develop thinking. The focus groups remained open and flexible and allowed researchers who were part of the physiotherapy student body to delve into experiences and develop themes. An independent researcher and registered physiotherapist interviewed the clinical supervisors and community-service therapists using semi-structured interview guides, as it was challenging to co-ordinate focus groups with the professionals in the respective groups (community-service physiotherapists and clinical supervisors). The relevant literature was reviewed and feedback from a pilot interview, as well as discussions with two experts in qualitative research methodology at the university, guided the process. A tape-recorder was used to capture narratives, while verbal nuances were recorded manually. The raw data were transcribed verbatim immediately after discussions and shared with the participants for verification.

\section{Data analysis}

Transcribed data were entered into NVivo 9 software (NVivo, USA) and read independently several times by researchers. Two teams of researchers derived themes and sub-themes. Two experts in qualitative methodology assisted in facilitating discussion around data and in attaining consensus among the researchers regarding themes and sub-themes. Methodological rigour was maintained through triangulation of data sources, member checking for veracity, peer debriefing and use of thick, rich descriptions. Furthermore, researcher bias was minimised by interviews conducted by peers, adding valued prompts by means of the data gathering. ${ }^{[11,13]}$

\section{Results}

Five overarching themes, i.e. preparedness for professional practice, institutional barriers, curriculum discrepancies, personal factors and recommendations for physiotherapy clinical education, were identified. Sub-themes were also derived from the triangulated data from the students, community-service physiotherapists and clinical supervisors.

Table 1 reflects the data of each group of participants, including age and gender.

The community-service therapists worked in environments spanning rural, urban and semi-rural settings. Five supervisors had $>5$ years of experience, 3 clinical supervisors had 1 - 5 years of experience, and 1 clinical supervisor had $>10$ years of experience.

Table 2 displays the themes and sub-themes that emerged from triangulation of the data.

\section{Themes}

1. Preparedness for professional practice and associated sub-themes, as well as perceived lack of graduate attributes for clinical practice, are described in the following narratives, together with illustrative quotes.

Some of the final-year students in this study felt unprepared to face clinical practice in their upcoming community-service year and thought that they were not sufficiently equipped to manage patients within certain fields of physiotherapy, such as paediatric care:

'We do get exposure but not in all areas ... other universities cover blocks like paediatrics.' (Student, Kaitlin)

Some students and the majority of the community-service physiotherapists believed that undergraduate clinical training provided a suitable foundation for clinical practice, patient care and clinical reasoning: 
Table 1. Biographical data and characteristics of participants $(N=40)$

\begin{tabular}{llllll} 
& \multicolumn{2}{c}{ Gender } & & \multicolumn{2}{c}{ Age group, years } \\
\cline { 2 - 5 } Participants & Male & Female & $\mathbf{2 0 - 2 9}$ & $\mathbf{3 0 - 3 9}$ & $\mathbf{4 0 - 4 9}$ \\
\hline Final-year students & 6 & 16 & 22 & - & - \\
Community-service physiotherapists & 3 & 6 & 9 & - & - \\
Clinical supervisors & 3 & 6 & 2 & 6 & -
\end{tabular}

Table 2. Summary of categories and themes

\section{Themes \\ Institutional barriers \\ Curriculum discrepancies}

Preparedness for professional practice

Personal factors

Recommendations for physiotherapy clinical education

\section{Sub-themes}

Perceived unpreparedness in exit year

Reflective preparedness for community service

Perceived lack of graduate attributes

Student/supervision ratio

Work overload

Time constraints

Site barriers

Disparity in curriculum design

Irrelevant theoretical content

Cohesive learning

Student/educator relationship

Personal perceptions

Student engagement

Communication breakdown

Theoretical bedside approach

Improved inclusive educational content

Improved curriculum design

Communication between stakeholders

Collaboration with clinicians
'It was a little difficult at first but I adapted quickly, physio knowledge [referring to undergraduate training] serves as a fair foundation. (Community-service physiotherapist, Anele)

The perceived lack of professional attributes, such as communication, was highlighted in the voices of the clinical supervisors:

'The dedication and calibre of students have changed and evolved in the past few years; students have a sense of entitlement and don't communicate properly.' (Clinical supervisor, Refilwe)

2. Institutional barriers. The quotes below reflect the barriers experienced by participants. The students reported that they were adequately supervised, but could benefit from smaller numbers of students per clinical supervisor:

'Compared to last year, it is now better to have smaller groups [referring to decreased number of students in blocks from $13-14$ to 6 - 7] ... I feel the smaller the number of students, the easier it is to supervise and I wish we had even fewer ... [giggles].' (Student, Sandy)

Work overload, time constraints and other site barriers posed further challenges to the clinical training platform, as mentioned by students and clinical supervisors:

'I feel like the qualifieds [physiotherapists at the hospitals] on clinical sites give us all too much work; it is because they have too much work to do.' (Student, Chris)
'Time is always a challenge, there is so much to learn and very little time to teach it all.' (Clinical supervisor, Angelique)

'Limited resources at the clinical sites like equipment is always a challenge, also sometimes you go through periods of insufficient patients on site.' (Clinical supervisor, Oupaman)

3. Curriculum discrepancies are reflected in illustrative quotes from the participants. The disparity in the curriculum design of the undergraduate programme is evident in echoed voices of students:

'Well, theory-wise I think for us Zulu-speaking students they should change that module to an English module ... irrelevant modules covered in first and second year.' (Student, Melusi)

Students indicated that some of the theoretical content taught in the programme was irrelevant:

'There are some theoretical parts [referring to the basic sciences] that we barely actually do apply!' (Student, Chante)

Cohesive agreement between theory and practical sessions seemed to be something students thought was neglected. They believed that there was a gap between what they were learning in the classroom and what they were expected to apply clinically at the healthcare settings when managing patients:

'I feel that sometimes they don't correlate what we learnt in class with what is expected in hospital; I couldn't apply it at the hospital.' (Student, Annie) 
4. Personal factors are illustrated by the quotes below. The relationship between student and educator seemed to be ill-defined and posed challenges to clinical education:

'Students feel we owe it to them to hand over all the information; it is not the norm but there is a trend to ask for things at any time and they don't want to meet you half way.' (Clinical supervisor, Chloe)

Perceptions, such as favouritism, seemed to be a challenge that faced students and hindered learning:

'We need to be treated fairly and equally. Supervisors need to stop this habit of having favourites, and level with us all.' (Student, Thomas)

Student engagement as active learners on the teaching platforms seemed to be another inhibitor in optimum clinical education:

'Students are not active participants of their own learning outcomes.' (Clinical supervisor, Refilwe)

Communication breakdown between stakeholders, e.g. clinical supervisors and clinical placement staff, posed a barrier to optimal learning:

'We would like the supervisors and the hospital physiotherapists to have the same stories ... during exam time we get so confused because of their contradicting ideas.' (Student, Xolani)

5. Recommendations for physiotherapy clinical education is the final reflection of the study results. A theoretical bedside approach was suggested as a way forward for students to bridge the gap between theory and clinical practice:

'It will also be nicer if the clinical supervisors would treat patients in front of you at the hospital.' (Community-service therapist, Nasreen)

Students echoed that a more comprehensive and inclusive undergraduate programme, including different aspects of physiotherapy, would benefit them:

'We need to have a sports block because the whole aspect of us choosing physiotherapy is to have different options when working, and more blocks to touch on paediatrics and strapping should be done in a module as well. (Student, Andiswa)

Students felt that the curriculum design could be improved by rearranging modules for fluidity, which would have a positive impact on clinical education and patient management:

'Some of the modules should be rearranged to help you, e.g. isiZulu should be brought into second or even third year to help us communicate better with our patients.' (Student, Thobile)

Students believed that the communication between stakeholders could be improved by tutorials to have a positive impact on clinical education:

'The lecturers (academic) should give the supervisors (clinical) tutorials about what we are learning so it [clinical education] will fit what we are learning in the classroom.' (Student, Moses)

Clinical supervisors were convinced that collaboration with clinicians on site would improve the clinical education framework:

'There needs to be buy-in and greater involvement with the clinicians (at placement sites), as they should be more involved in clinical education. (Clinical supervisor, Africa)

\section{Discussion}

Clinical education is essential for the training of physiotherapists to prepare them for autonomous practice in their future careers as healthcare professionals. The evidence in a SA context is scarce - insight was gleaned from a broader international framework of the literature regarding various approaches to and perceptions of clinical education for physiotherapy students and their clinical training.

It is believed that students learn the technical skills, as well as social and attitudinal competencies, to manage patients in their communities during their clinical education in undergraduate programmes. ${ }^{[5,6]}$ Final-year physiotherapy students who participated in the focus group discussions felt unprepared to manage patients and apprehensive to start their communityservice practice year. According to Roman and Dison, ${ }^{[14]}$ SA universities are currently facing a lack of student preparedness, which is attributed to an array of factors, including the multilingual needs of the students and the large intake of students into university programmes. However, according to Ramli et al., ${ }^{[15]}$ students experienced anxiety towards initial exposure during clinical placements, but later developed a sense of confidence in their professional competencies. Interviews with community-service therapists revealed that they only experienced retrospectively that the undergraduate training adequately prepared them for community service. Students' undergraduate education must equip them with skills for continuous reflective practice. ${ }^{[16]}$ Mostert-Wentzel et al., ${ }^{[17]}$ in an appreciative inquiry into experiences of community-service physiotherapists in SA, advocated for reflection with regard to clinical learning in undergraduate training that could enhance clinical practice. If students were to critically reflect on the clinical education experienced during their placements, this should impact on their learning and the actions that emanate from it, which will contribute to deepened learning. ${ }^{[15]}$ Clinical supervisors need strategies to stimulate reflection on learning at an undergraduate level to enhance clinical education and learning in graduate programmes. ${ }^{[5]}$ Reflective diaries and learning contracts are means used to enhance reflective practice, aid in developing thought and create change in students' personal learning methodologies. ${ }^{[15,18]}$ Mostert-Wentzel et al. ${ }^{[17]}$ suggested that discussions and presentations could form part of reflections for clinical education. Blended learning and online and face-to-face teaching are also reported to improve reflective skills of physiotherapy students. ${ }^{[16]}$

The healthcare system in SA is faced with resource constraints, which often inhibit the implementation of models of healthcare that address the burden of disease in the country. ${ }^{[19]}$ These constraints include healthcare staff shortages and infrastructural limitations. ${ }^{[20]}$ Similar constraints have hindered teaching and learning as perceived by participants in the study, as they felt that the shortage of staff, time constraints and lack of equipment influenced their clinical learning experience. Congruently, Parry and Brown, ${ }^{[21]}$ in their study set at physiotherapy teaching institutions in the UK, stated that challenges for both teaching and assessment in communication strategies for physiotherapy students were a lack of resources, time, staffing and expertise. Stiller et al., ${ }^{[8]}$ seeking insight into clinical education models in Australia, agreed that healthcare staff are also faced with patient demands, administration and other clinical duties. Students further emphasised that their large numbers compared with those of clinical supervisors at clinical settings impacted on their learning. They believed that fewer students in clinical placements were preferable, as it improved their learning. A study conducted at the same setting revealed that a large number of students being 
supervised by a limited number of clinical supervisors posed challenges to clinical feedback and supervision within clinical education platforms. ${ }^{[22]}$ However, a study by Sevenhuysen et al. ${ }^{[23]}$ comparing traditional clinical education (similar to the context of the current study) with a peer-assisted learning model of allied health science students in Australia, found that although the latter model diminished the clinical supervisors' workload, students and educators preferred the traditional approach.

Participants in this study echoed that the curriculum needs to be reviewed owing to disparities, such as irrelevant content and lack of fluidity in the design. It is imperative for students to transform theory into practice. Ramklass, ${ }^{[22]}$ in her study at the same setting, found that the design influences the delivery and quality of the undergraduate physiotherapy programme. Another health sciences study at the same institute found that harmonious theoretically and clinically based teaching is vital for adequate clinical preparation of undergraduate health professions students. ${ }^{[24]}$ Students in this study agreed that there should be more cohesive learning and fluidity in the curriculum design, which is in keeping with a study involving physiotherapists graduating from the same university, who indicated that the absence of theoretical knowledge prior to clinical practice was perceived as a negative attribute of the programme. ${ }^{[22]}$ Physiotherapy students at a university in the Western Cape, SA, felt adequately prepared for clinical practice, which was attributed to a thorough review and alignment of the physiotherapy curriculum. ${ }^{[25]}$ Health science curricula necessitate review and revision to accommodate the dynamic climate of healthcare in SA. ${ }^{[6]}$

The relationship between all stakeholders in the learning process in clinical education approaches needs to be collaborative to achieve success. The students in the study perceived that favouritism was inhibiting their learning process. Students believed that clinical supervisors were paying more attention to some of their peers, which needs to change for improved learning. Students also believed that communication between stakeholders, i.e. clinical supervisors and hospital clinical staff, was lacking. A study in Ireland on barriers and facilitators to a physiotherapy education approach supported collaboration between all stakeholders in the education framework, which facilitated learning and contributed to adequately prepared students. ${ }^{[26]}$ According to Lo et al., ${ }^{[27]}$ the partnership and working together of the healthcare student and the clinical supervisor are essential to offer an environment of effective learning of technical skills, core competencies and ethical and social integration into clinical practice. Furthermore, Olsen et al ${ }^{[28]}$ indicated that clinical supervisors are role models and responsible for the students in clinical placement areas; they are also the main information source for students. There was inconsistency in what was taught in the classroom and what clinical staff at healthcare settings communicated. In a study by De Witt et al., ${ }^{[29]}$ clinicians involved in education of occupational therapy students felt that they were not adequately prepared to teach undergraduate students and feared judgement by the students. Talberg and Scott ${ }^{[25]}$ conceded that lack of preparedness emerges when what is taught in the classroom does not translate into clinical practice. The ongoing communication between the academic institution and the clinical placement area offers an enabling environment for students' clinical education. ${ }^{[26]}$

The clinical supervisors in this study were challenged by their perception of students, who they believed were not playing a pivotal active role in their learning and placed gratuitous pressure and demands on the supervisors.
Strohschein et al. ${ }^{[30]}$ stated that both students and educators should engage in an 'intentional, structured process of changing roles during the course of the clinical education process', meaning a deliberate change in roles to facilitate understanding and improve learning. They also believed that the core competencies, such as communication, collaboration and reflection, formed an integral part of effective learning in clinical education frameworks. Rowe et al. ${ }^{[16]}$ found that a 'mutually beneficial' relationship between students and clinical supervisors should be sought to improve learning.

The success of the current education platform, as recommended by participants, hinged on improved teaching strategies, such as more bedside tutorials, improved curriculum design and better communication and collaboration among stakeholders, including clinical site staff and clinical supervisors. In Ernstzen et al.'s ${ }^{[5]}$ article, valuable learning opportunities as perceived by physiotherapy students in clinical settings included demonstration of management of patients, discussion, feedback and assessment (both formative and summative). However, the demonstrations of student-led patient management were more valuable than those that were teacher led, as students believed they were central and actively engaged. This paradigm shift is essential for clinical education as the student takes 'centre stage. The student-centred strategy leads to learning by discovery, co-creation of knowledge, and direct and reflective learning. ${ }^{[5]}$ Furthermore, as highlighted in the recommendations, communication is a key competency for graduates. It is not merely fundamental for clinical practice and patient management, ${ }^{[31]}$ but also integral for effective team collaboration and a holistic approach to rehabilitation. ${ }^{[25]}$

\section{Conclusion}

Clinical education frameworks are dependent on stakeholder involvement and collaboration, teaching and learning opportunities experienced by students, as well as culture at the clinical placement area. ${ }^{[5,31]} \mathrm{A}$ deeper understanding of how students and educators interpret the clinical education framework is important in the delivery of education and how to improve it to address the needs of a diverse healthcare context. ${ }^{[31,32]}$ This study aimed to understand the perspectives of students, past students who were doing community service at the time of the study and clinical supervisors of a physiotherapy clinical education framework at a university in SA. The study yielded results that support the ongoing review and alignment of the physiotherapy curriculum, as well as improved collaboration between all stakeholders. Moreover, the transformative learning shift needs to be in the forefront, as students move to the focal point of their own learning. In a Lancet $^{[33]}$ article, a commission of 20 professional and academic leaders from various countries rallied together to develop a common vision for health science, leaving the issues of tribalism and national boundaries aside. The collaborators advocated for a generation of graduates who are 'systems based', who should influence health systems and have core competencies that are locally driven but have global relevance. ${ }^{[33]}$ The commission also acknowledged that specific institutional and instructional reforms are necessary and could be led by transformative learning. ${ }^{[33]}$ The adoption and shift toward transformative learning in physiotherapy curricula across SA could enhance socialisation of students around values and core competencies, resulting in leaders who are critical engagers and creative thinkers. ${ }^{[33]}$ Further discussions and research are recommended to begin institutional debate on feasible approaches to incorporate transformative learning into our education systems. 
Acknowledgements. The authors would like to thank the participants for their time and valuable input with regard to the article. We also thank the participating students for their honest responses and contribution to the study.

Author contributions. VC, SM, SC and NP: conceptualised the study and recruited the research students to assist with the project. VC and SM: collected the data together with the students. VC, SM, SC and NP: reviewed, analysed and wrote the article.

\section{Funding. None.}

Conflicts of interest. None.

1. World Confederation for Physical Therapists. http://www.wcpt.org/search/node/role\%20of\%20physiotherapy (accessed 15 May 2017)

2. Health Professions Council of South Africa. http://www.hpcsa.co.za/PBPhysiotherapy (accessed 15 May 2017). 3. University of KwaZulu-Natal. http://physiotherapy.ukzn.ac.za/Homepage.aspx (accessed 15 May 2017).

4. Moore A, Morris J, Crouch V, Martin M. Evaluation of physiotherapy clinical educational models: Comparing 1: 1, 2: 1 and 3: 1 placements. Physiotherapy 2003;89(8):489-501. https://doi.org/10.1016/S0031-9406(05)60007-7 5. Ernstzen DV, Bitzer E, Grimmer-Somers K. Physiotherapy students' and clinical teachers' perceptions of clinical 7. Ernstzen DV, Bitzer E, Grimmer-Somers K. Physiotherapy students' and clinical teachers' perceptions of clinica
learning opportunities: A case study. Med Teach 2009; (3):e102-e105. https://doi.org/10.1080/01421590802512870 6. Krause MW, Viljoen MJ, Nel MM, Joubert G. Development of a framework with specific reference to exit-level 6. Krause MW, Viljoen M), Nel MM, Joubert G. Development of a framework with specific reference to exit-level
outcomes for the education and training of South African undergraduate physiotherapy students. Health Policy outcomes for the education and training of South African undergraduate physiotherapy students. Health Policy 2006;77(1):37-42. https://doi.org/10.1016/j.healthpol.2005.07.015

7. Lekkas P, Larsen T, Kumar S, et al. No model of clinical education for physiotherapy students is superior to another: A systematic review. Austr J Physio 2007;53(1):19-28. https://doi.org/10.1016/S0004-9514(07)70058-2 8. Stiller K, Lynch E, Phillips AC, Lambert P. Clinical education of physiotherapy students in Australia: Perception of current models. Austr J Physio 2004;50(4):243-247. https://doi.org/10.1016/S0004-9514(14)60114-8

9. Govender P, Chetty V, Naidoo D, Pefile N. Integrated decentralized training for health professions education at the University of KwaZulu-Natal, South Africa: Protocol for the I-DecT Project. JMIR Res Protoc 2018;7(1):e19. https://doi.org/10.2196/resprot.7551

10. Aberdeen T, Yin RK. Case study research: Design and methods. Can J Action Res 2013;14(1):69-71.

11. Baxter P, Jack S. Qualitative case study methodology: Study design and implementation for novice researchers. Qual Rep 2008;13(4):544-559.

12. Patton MQ. Qual Res. Online library: John Wiley, 2005. https://doi.org/10.1002/0470013192.bsa514

13. Creswell JW, Miller DL. Determining validity in qualitative inquiry. Theory Pract 2000;39(3):124-130. https:// doi.org/10.1207/s15430421tip3903_2

14. Roman NV, Dison A. Relationship between student preparedness, learning experiences and agency: Perspectives from a South African university. Afr J Health Professions Educ 2016;8(1):30-32. https://doi.org/10.7196/AJHPE.2016. v8i1.490

15. Ramli A, Ruslan AS, Sukiman NS. Reflection of physiotherapy students in clinical placement: A qualitative study Sains Malaysiana 2012;41(6):787-793.

16. Rowe M, Frantz J, Bozalek V. The role of blended learning in the clinical education of healthcare students: A systematic review. Med Teach 2012;34(4):e216-e221. https://doi.org/10.3109/0142159X.2012.642831
17. Mostert-Wentzel K, Frantz I van Rooijen AJ. A model for community physiotherapy from the perspective of newly graduated physiotherapists as a guide to curriculum revision. Afr J Health Professions Educ 2013;5(1):19-25. https://doi.org/10.7196/AJHPE.203

18. Ramli A, Joseph L, Lee SW. Learning pathways during clinical placement of physiotherapy students: A Malaysian experience of using learning contracts and reflective diaries. J Educ Eval Health Prof 2013;10:6. https://doi org/10.3352/jeehp.2013.10.6

19. Chetty V, Hanass-Hancock J. The need for a rehabilitation model to address the disparities of public healthcare for people living with HIV in South Africa: Opinion papers. Afr J Disabil 2015;4(1):1-6. https://doi.org/10.4102/ ajod.v4i1.137

20. Cobbing S, Chetty V, Hanass-Hancock J, Jelsma J, Myezwa H, Nixon SA. The essential role of physiotherapists in providing rehabilitation services to people living with HIV in South Africa. S Afr J Physio 2013;69(1):22-25. https://doi.org/10.4102/sajp.v69i1.368

21. Parry RH, Brown K. Teaching and learning communication skills in physiotherapy: What is done and how should it be done? Physiotherapy 2009;95(4):294-301. https://doi.org/10.1016/. physio.2009.05.003

22. Ramklass $\mathrm{S}$. The clinical education experience of student-physiotherapists within a transformed model of healthcare. Internet J Allied Health Sci Pract 2013;11(2):4.

23. Sevenhuysen S, Skinner EH, Farlie MK, et al. Educators and students prefer traditional clinical education to a peer-assisted learning model, despite similar student performance outcomes: A randomised trial. J Physio 2014;60(4):209-216. https://doi.org/10.1016/j.jphys.2014.09.00

24. Naidoo D, van Wyk J. Fieldwork practice for learning: Lessons from occupational therapy students and thei supervisors. Afr J Health Professions Educ 2016;8(1):37-40. https://doi.org/10.7196/AJHPE.2016.v8i1.536

5. Talberg H, Scott D. Do physiotherapy students perceive that they are adequately prepared to enter clinica practice? An empirical study. Afr J Health Professions Educ 2014;6(1):17-22. https://doi.org/10.7196/AJHPE.219

26. McMahon S, Cusack T, O'Donoghue G. Barriers and facilitators to providing undergraduate physiotherapy clinical education in the primary care setting: A three-round Delphi study. Physiotherapy 2014;100(1):14-19. https://doi.org/10.1016/j.physio.2013.04.006

27. Lo K, Osadnik C, Leonard M, Maloney S. Differences in student and clinician perceptions of clinical competency in undergraduate physiotherapy. NZJ Physio 2015;43(1):11-15. https://doi.org/10.15619/NZJP/43.1.02

28. Olsen NR, Bradley P, Lomborg K, Nortvedt MW. Evidence based practice in clinical physiotherapy education A qualitative interpretive description. BMC Med Educ 2013;13(1):52. https://doi.org/10.1186/1472-6920-13-52

29. De Witt P, Rothberg A, Bruce J. Clinical education of occupational therapy students: Reluctant clinical educators. S Afr J Occupational Ther 2015;45(3):28-33. https://doi.org/10.17159/2310-3833/2015/v45n3/a6

30. Strohschein J, Hagler P, May L. Assessing the need for change in clinical education practices. Phys The 2002;82(2):160-172. https://doi.org/10.1093/ptj/82.2.160

31. Ernstzen DV, Bitzer E, Grimmer-Somers K. Physiotherapy students' and clinical teachers' perspectives on bes clinical teaching and learning practices: A qualitative study. S Afr J Physio 2010;66(3):25-31. https://doi.org/10.4102 sajp.v66i3.70

32. Chan DS. Combining qualitative and quantitative methods in assessing hospital learning environments. Int Nurs Stud 2001;38(4):447-459. https://doi.org/10.1016/S0020-7489(00)00082-1

3. Frenk J, Chen L, Bhutta ZA, et al. Health professionals for a new century: Transforming education to strengthe health systems in an interdependent world. Lancet 2010;376(9756):1923-1958. https://doi.org/10.1016/S01406736(10)61854-5

Accepted 29 August 2017 